\title{
Characterizing and Indexing Regolith Materials Using Geochemistry towards Hidden Mineral Anomaly Delineation: A Case Study of Savannah Region of NW Ghana
}

\author{
Emmanuel Arhin", Musah S. Zango, Raymond Kazapoe \\ Department of Earth and Environmental Sciences, Faculty of Applied Sciences, University for Development Studies, P.O. Box 24, \\ Navrongo, Ghana
}

Copyright(C2017 by authors, all rights reserved. Authors agree that this article remains permanently open access under the terms of the Creative Commons Attribution License 4.0 International License

\begin{abstract}
As the discovery rate of world-class gold deposits continues to decline, increased attention is focused on geochemical exploration methods designed for regolith-dominated terrains. This involves mapping the regolith and classifying the mapping units into different regolith classes on the basis of weathering and geomorphic histories. The challenges of identifying some regolith mapping units in the field by novice in regolith mapping require the characterization and indexing of regolith from major element geochemistry in the regolith profiles. XRF analytical method was used to measure the weight $\%$ of the major oxides in regolith samples. The metal weight $\%$ of $\mathrm{Mg}, \mathrm{K}$ and $\mathrm{Al}$ were calculated from their oxides and were normalized relative to immobile $\mathrm{Al}$ calculated from its oxide. The plot of $\mathrm{Mg} / \mathrm{Al}$ and $\mathrm{K} / \mathrm{Al}$ identified the regolith of the study area to consist of 137 transported clays, 4 ferruginous sediments or ferricrete, 2 lateritic duricrust and 4 saprolites. Plot of $\mathrm{Mg} / \mathrm{Al}$ and $\mathrm{K} / \mathrm{Al}$ highlighted the compositional variability of the regolith samples and refute the notion of the homogeneity of all the sampled materials in the area. The study thus recognized $\mathrm{Mg} / \mathrm{Al}$ versus $\mathrm{K} / \mathrm{Al}$ plots to be used in supporting field identification of regolith mapping units particularly in complex regolith terrains of savannah regions of Ghana and in similar areas where geochemical exploration surveys are being carried out under cover.
\end{abstract}

Keywords Regolith, Weathering Histories, Savannah Region, Ratio Plot, Ghana

\section{Introduction}

Apart from mineral deposits with mineralisation readily discernible in rock outcrops by identification of any mineral anomaly has to be carried out by sampling the regolith. Regolith as known to mineral exploration geologists represents materials overlying the coherent bedrock and contains unconsolidated and secondarily cemented materials [1-3]. It thus forms the oxidizing environment of the earth's landscape and can have simple and/or complex weathering histories depending on the climatic and the anthropogenic activities. Superficially the surface regolith materials look alike and require experts in regolith mapping to distinguish the differences between the various domains in regolith complex terrains. From $[1,2]$ the regolith is found atop the bedrock and can host potential mineral anomalies of the deep seated orebody or an expression of the deep seated or hidden mineralisation can be detected in them. The climate everywhere do impact on the hydrological, weathering and geomorphic processes hence producing mosaic of different regolith units, changing rapidly and thus affecting the geochemical expressions in the surface regolith. Butt and Zeegers [4] show a wide range of materials within a single regolith profile or toposequence with great variations in mineralogical and chemical composition, as well as variations in fabric and origin. Arhin et al. [1] confirmed the complexities of regolith, particularly in the savannah regions of Ghana when they recognized the ferruginous regolith regime that consists of lateritic duricrust, lateritic gravel, ferruginous duricrust or ferricrete and all ferruginous materials whose origins cannot be identified easily in the field and generally called cuirasse by the French. 

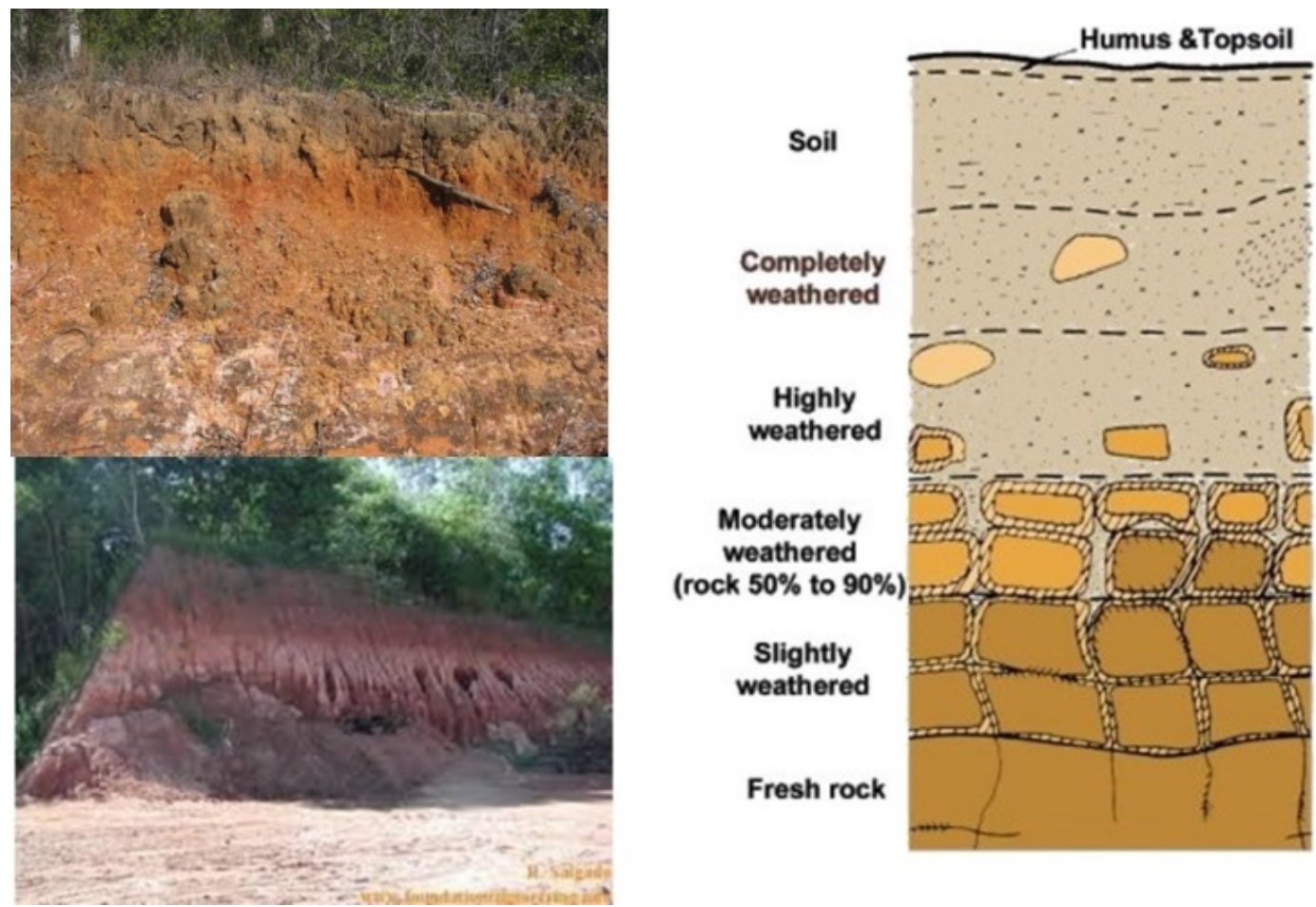

Figure 1. Images of residual regolith formed under simple weathering processes in the study area
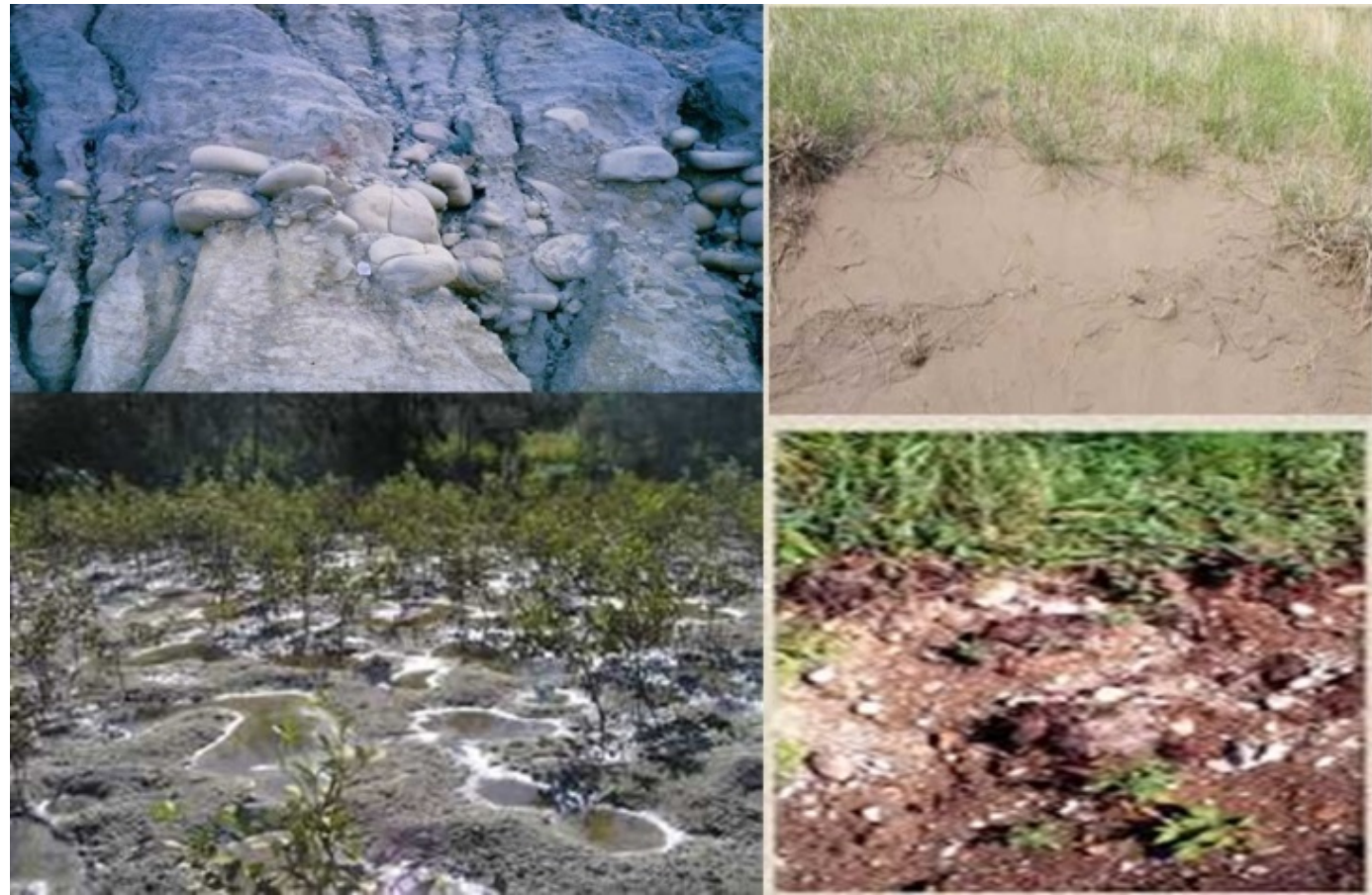

Figure 2. Images of transported regolith formed under complex weathering processes in the study area 

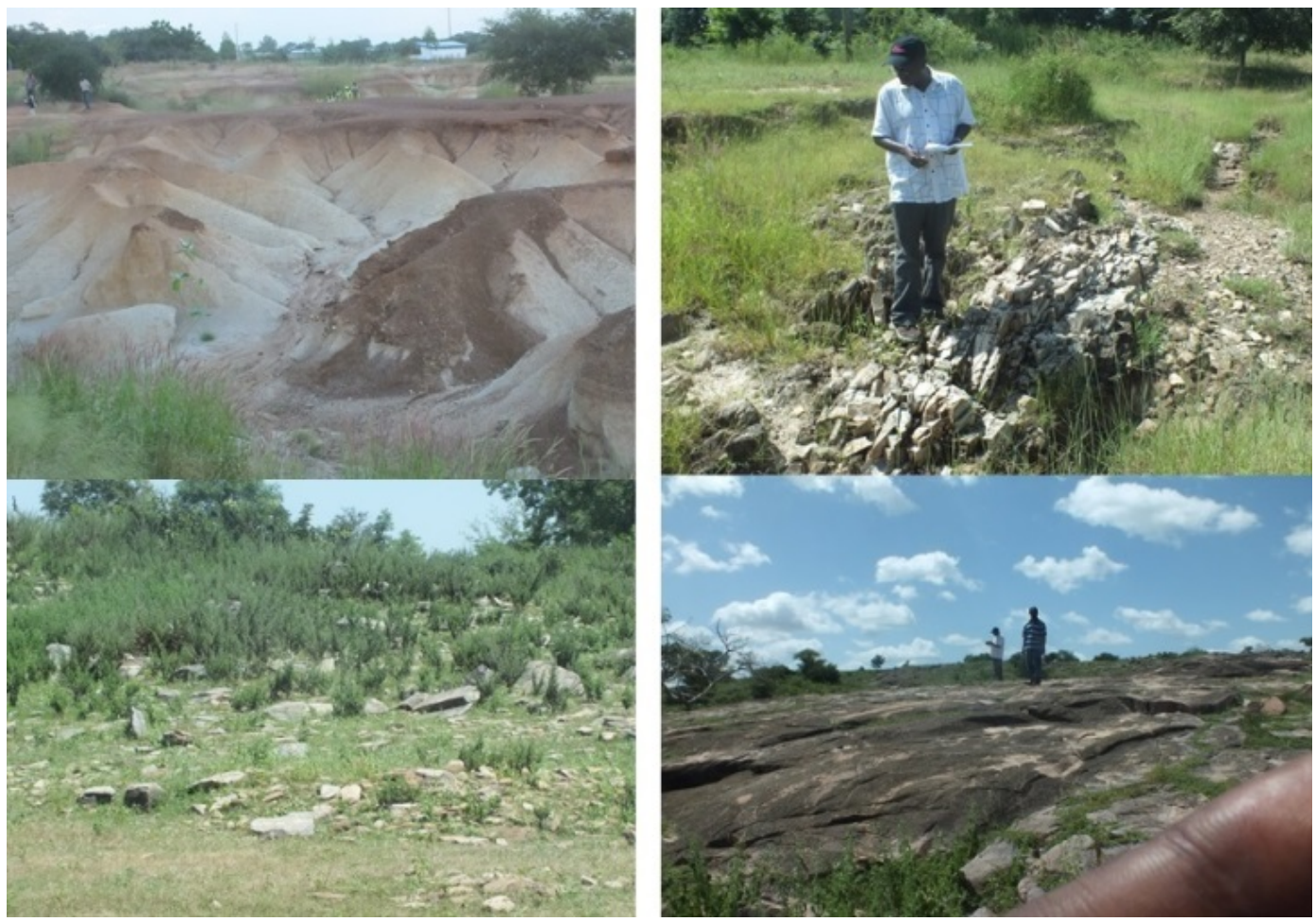

Figure 3. Images of erosional regolith formed under complex weathering processes in the study area

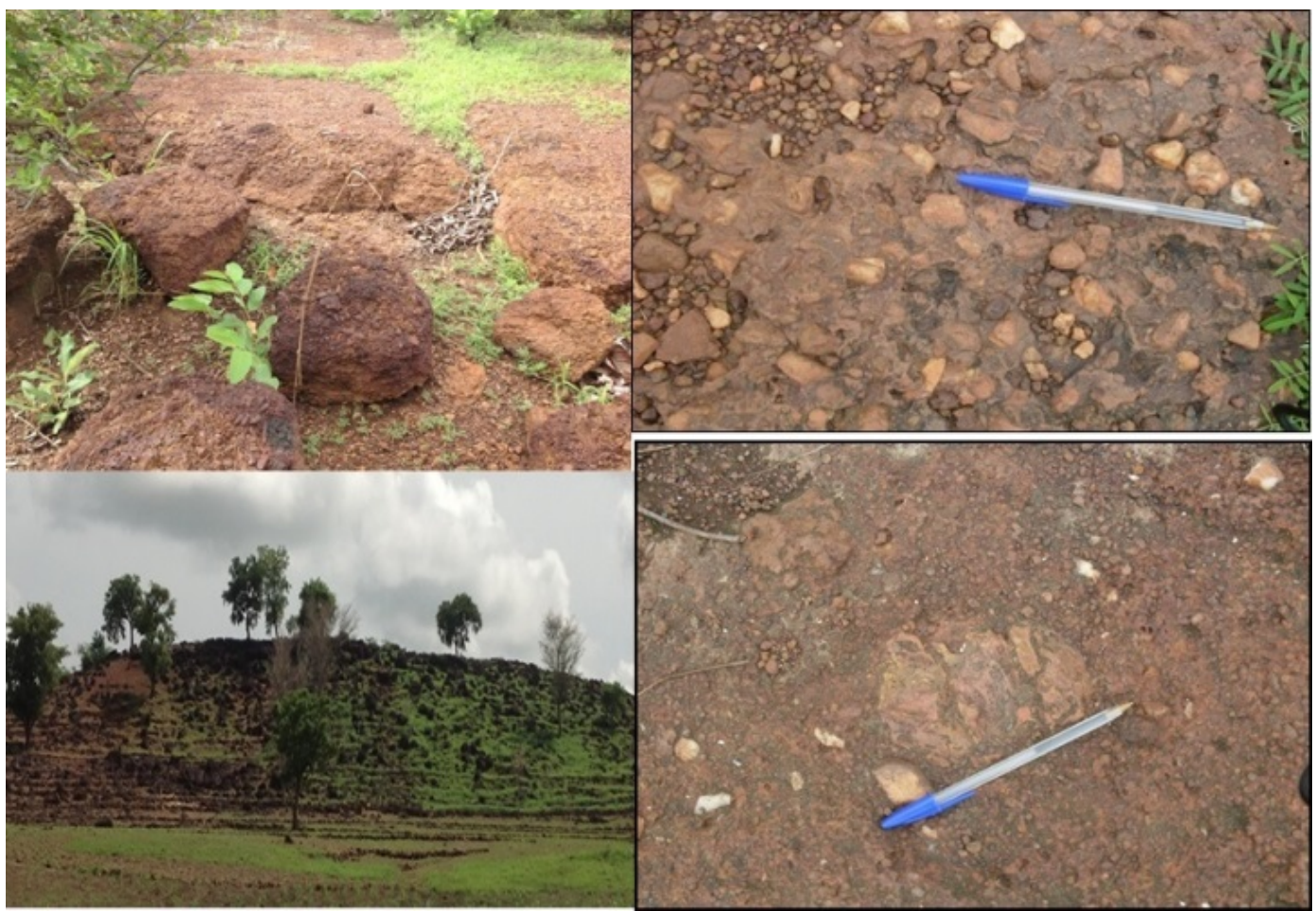

Figure 4. Images of ferruginous regolith formed under landscape and regolith evolution processes in the study area 


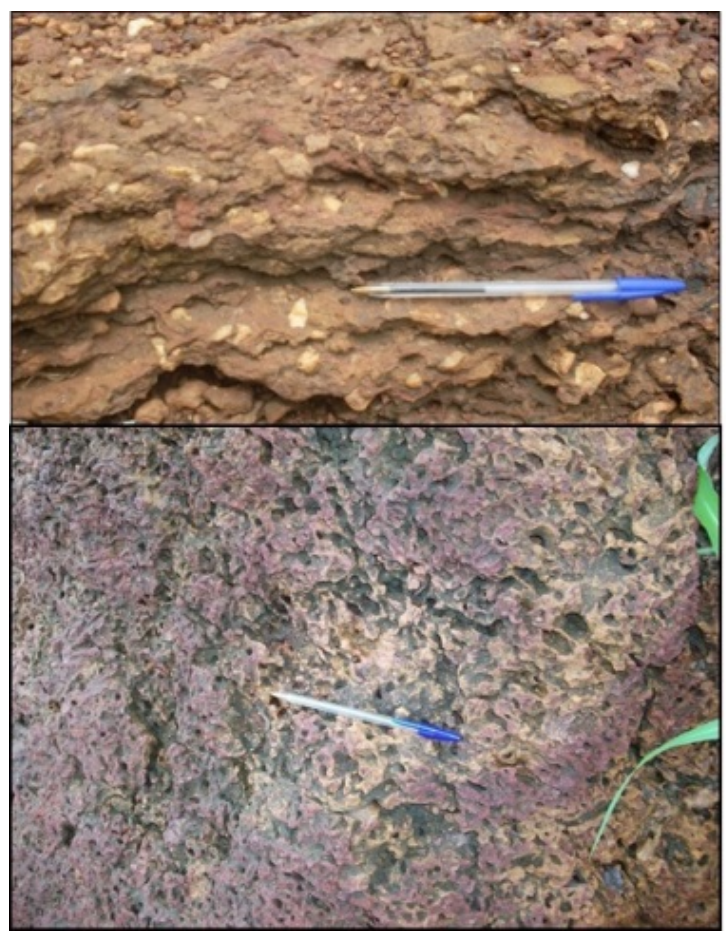

\section{Ferruginous Duricrust-Ferricrete}

\section{Lateritic Duricrust- Characterised by Equigranular groundmass}

Figure 5. Images of transported and residually formed ferruginous regolith in the study area (Ferruginous duricrust representing transported laterite and Lateritic duricrust considered as residual laterite)

The anxiety of many mineral exploration geologists is to identify the differences and map correctly the various regolith classes in order to detect the hidden mineral deposits. The declining rates of world-class mineral deposit discoveries in less complex regolith terrains has resulted in exploration companies broader attention to devise geochemical exploration methods suitable for regolith-dominated terrains [2-4]. However, there is a caveat that some regolith mappers in Africa had some misleading classifications for some duricrusts (cuirasses), whose origin is now disputed [5, 6]. Arhin and Nude [2] believe the declining trend of mineral discoveries particularly in West Africa is due in part to the continuous evolution of the regolith and landforms in which the minerals occur in the surface environment. Therefore for sustainability of mineral industries in regolith-dominated terrains proper identifications of the regolith classes are essential for correct surface geochemical interpretations. Exploration companies have loads of multi element data that can be processed, characterized and used to identify the various regolith regimes. As noted by Arhin and Zango [7] determining the regolith mineral composition could be major ways to characterize different regolith materials but the regolith grain fineness hampers their use. Nonetheless in consideration that rock is an aggregate of minerals the geochemistry of the available primary minerals released at the weathering front to the end-regolith-stage during weathering can be used as a broad surrogate for the regolith mineralogy. On the basis of these ratio plots of $\mathrm{K} / \mathrm{Al}$ and $\mathrm{Mg} / \mathrm{Al}$ measured by XRF analytical method that compares two mobile elements with a relatively immobile element from regolith samples is used to provide a simple index to characterize and identify the different regolith materials in some areas in the savannah regions of NW Ghana.

\section{Location, Physiographic Settings and Geology}

\subsection{Location and Physiographic Settings}

The research area is located in the Lawra Birimian belt in northern Ghana $700 \mathrm{~km}$ northwest of Accra, the national capital and the climate is savannah with annual rainfall of about 600-1200 $\mathrm{mm}$ (Webber, 1996). The area has one rainy season which starts slowly from March and peaks in August after which there is a sharp decrease after October. Monthly average rainfall is $986 \mathrm{~mm}$. Temperatures are regularly high with monthly averages at $28.6{ }^{\circ} \mathrm{C}$ which drop to a minimum of $26.4{ }^{\circ} \mathrm{C}$ at the peak of the rainy season in August and to a maximum of $40{ }^{\circ} \mathrm{C}$ in April [8]. However the diurnal temperature changes has been noted to exceed monthly variations in the area [8] confirming the known knowledge in the tropics about the diurnal temperature variations. The total evaporation of $2050 \mathrm{~mm}$ recorded for the area exceeds the annual rainfall more than two fold. These conditions are favourable for lateritization processes to form laterites [4, 9] and will contribute to the rapid changing regolith [1]. 


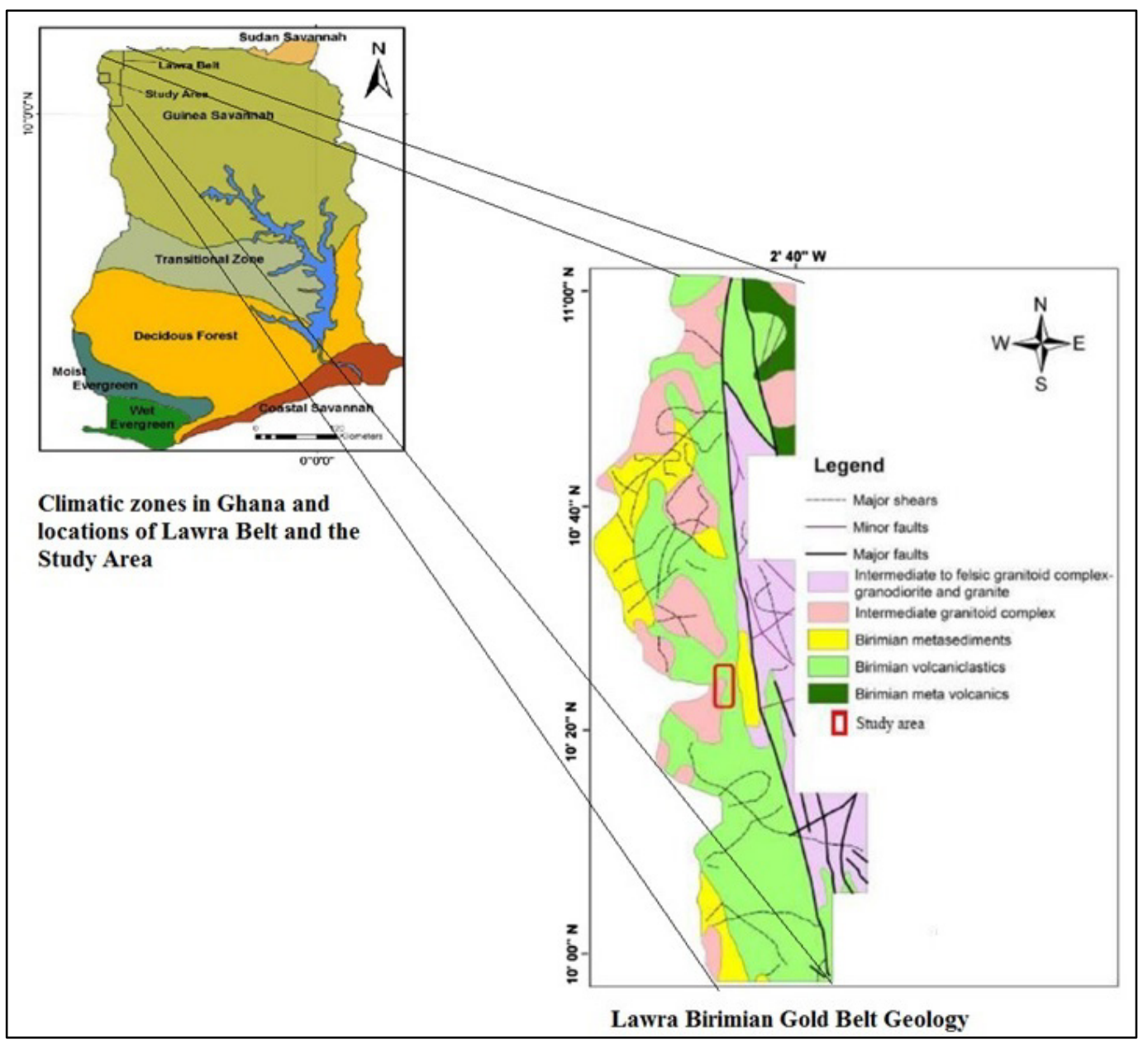

Figure 6. Regional geology and location of the study (Ghana Geological Survey, 2010)

\subsection{Geology}

The regional geology is shown in Fig. 6 with metamorphosed lavas and pyroclastic rocks containing basalts, andesite, rhyolites, dolerites with gabbroic intrusive particularly characterizing mostly the eastern and the southern block of the area [10-14]. The western block consists mainly of metasedimentary suite of rocks. Patches of dark grey to greenish grey, weakly foliated mafic extrusive volcanic rocks that are partially assimilated with melanocratic relicts (xenoliths) in close association with granitoids are not uncommon at the western portion of the study area [2]. The metasedimentary units consist of phyllite, sericite-schist and metagraywacke that are locally intruded by felsic and mafic dykes. Intruding the metamorphosed lavas, the pyroclastic rocks and the metasedimentary rock suites are Migmatite bodies and porphyritic granitoids that have generally been classified into two broad categories. Hornblende-rich intrusive varieties have close association with the metavolcanic rocks and these are known as 'Dixcove' or 'Belt' Type; and the mica-rich intrusive rocks that tend to border the volcanic belt and intrude the metasedimentary rocks are referred to as Basin-Type Granitoids. The belt-granitoids are discrete-discordant mafic granitoids whilst the basin-granitoids are large felsic, concordant and syntectonic batholiths commonly banded and foliated. The basin granitoids are two-mica potassic types, containing both biotite and muscovite, with the biotite dominating $[15,16]$.

\subsection{Regolith}

The regolith of the study area (Fig. 7) is characterized by a deep weathering profile with a pattern-less spatial distributions of relict, erosional and depositional regolith plus surfaces affected by widespread lateritization. The general picture of the regolith is a mosaic of surface veneer of pisoliths, laterite caps and residuum, saprolite and bedrock exposures and depositional cover of exotic origin. The low-lying areas are characterized by sheet wash deposits and the gently-slopes have blankets of thin layers of colluvium, which are interspersed with alluvial deposits often at the base of the slopes. The upland areas are generally marked by scree that decreases in fragment size down-slope. Topography is generally low, undulating with isolated hills at the western side. On the contrary topographic high landscapes represent the eastern side [2]. Most parts of the landscape rises gently between elevations of $100 \mathrm{~m}$ and $250 \mathrm{~m}$ above mean sea level [1]. 


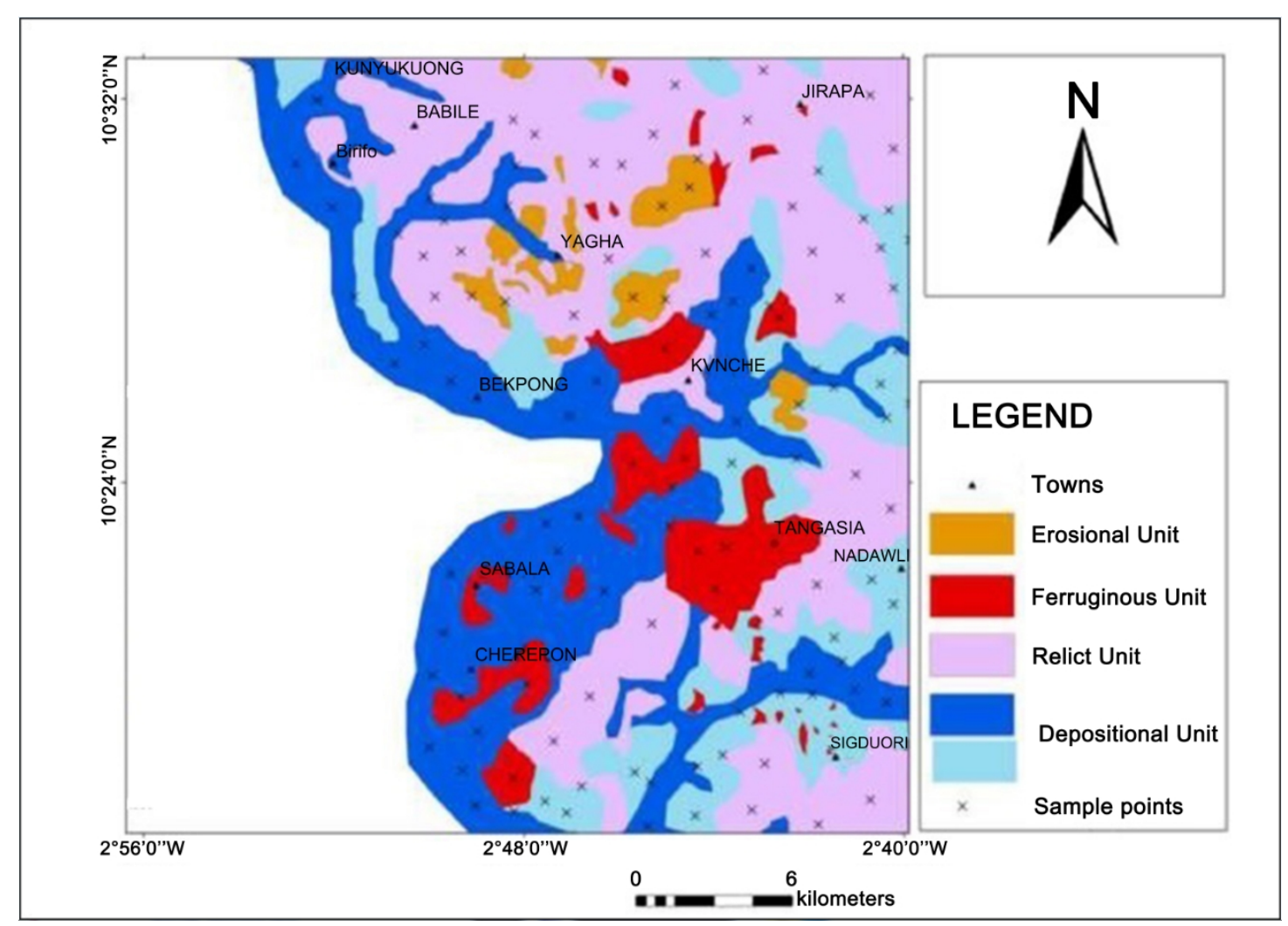

Figure 7. Regional regolith map (1:50000; Arhin et al. 2015)

The Fe-oxide cementing landscapes or the ferruginous landscapes are characterized occasionally by hardpans but often are covered by thin layers of pisolithic soils. Laterites formed from residual regolith units occur on the isolated high rises. These laterites generally show equigranular groundmass. The depositional regolith and ferricrete are found generally at low-lying areas and around drainage catchment areas. Ferricrete or transported laterites are widespread at high and low pediments and sometimes in stream basins [2]. Characterizing the ferruginous duricrust and ferricrete are the unique rounded and sub-rounded lithic and quartz pebbles cemented together with polymictic regolith matrix by clay minerals and $\mathrm{Fe}$-oxide. There are some that have older laterite with high degree of $\mathrm{Fe}$-oxide surface crust degraded likely from the intermediate relief surface and got embedded in an immature or younger laterite at high glacis surfaces. Notwithstanding the Fe-oxide-rich soils certain areas are overlain by indurated saprolite which usually appears hardened like a hardpan. The erosional areas that expose the saprolite are generally uncommon.

\section{Materials and Methods}

The methods used involved field and laboratory work. A regolith landform unit base map was used to plan the sampling points. The predetermined sampling points covered the ferruginous, relict, erosional and depositional regimes. The sampling locations are shown in Figure 7.
The samples were collected from a circular hole of $30 \mathrm{~cm}$ nominal diameter; dug up to $30 \mathrm{~cm}$ depth from the land-surface. A total of 154 regolith samples were collected in a non-gridded pattern and their locations recorded using a GPS receiver device. Breakdown of samples collected from the different regolith-landform unit classes were 25 from ferruginous regime; 34 from relict regime; 20 from erosional regime and 60 from depositional regime. Additional 15 samples representing residual samples (i.e. relict and erosional samples) were collected from termite mounds located generally at the depositional and ferruginous regolith terrains. The collected samples were sieved to $<125 \mathrm{~mm}$ size fractions for the reason that metal elements concentrate well in fine fractions. The sieved samples were analyzed by the XRF method at the Ghana Geological Survey Department.

In this study the XRF method was used to determine the major elements on fused glass beads prepared from ignited powders whereas the trace elements were analyzed on $32 \mathrm{~mm}$ diameter pressed powder pellets produced from mixing $7 \mathrm{~g}$ fine ground sample powder with 12-15 drops of $7 \%$ Mioviol 8-88 solution pressed at 10 tons per square inch. The pellets were placed in XRF machine connected to a computer with the Spectro X-lab software that records the concentrations of the major and trace elements in the samples. Precision and accuracy of the analytical data were obtained by repeating the analysis and comparing loss of ignition (LOI) of original analysis to the recalculated loss of ignition of repeated analysis. Four 
reference materials subsampled from the same material and given different sample numbers BCS 376, BCS 375, WS-1 and BH-1 were also inserted to check on analytical accuracy. The precision of the analytical data was also evaluated by comparing the nearness of the original results obtained from the fused glass beads and the re-calculated results of the major elements.

\section{Results}

Results of major oxides are shown in Table 1. The calculated ratios of $\mathrm{K} / \mathrm{Al}$ and $\mathrm{Mg} / \mathrm{Al}$ from the all the regolith samples are presented in Table 2. Figure 8 also represents the ratio plot of $\mathrm{K} / \mathrm{Al}$ and $\mathrm{Mg} / \mathrm{Al}$ which identifies and indexed the various regolith samples into specific regolith domains. The collected samples were taken from superficial regolith materials which at first instance look similar in texture and nature and could be considered as having the same weathering and geomorphic histories exceptions being the hardpan covered terrains. The quality of the analytical data for the mobile and relatively immobile oxides $\mathrm{Al}_{2} \mathrm{O}_{3}, \mathrm{MgO}$ and $\mathrm{K}_{2} \mathrm{O}$ is shown in Figure 9.

Table 1. Results of major oxides in regolith samples

\begin{tabular}{|c|c|c|c|c|c|c|c|c|c|c|c|c|}
\hline \multicolumn{13}{|c|}{ XRF Results for major oxides in regolith samples } \\
\hline ead & $\mathrm{SiO}_{2}$ & $\mathrm{TiO}_{2}$ & $\mathbf{A l}_{2} \mathbf{O}_{3}$ & $\mathrm{Fe}_{2} \mathrm{O}_{3}$ & MnO & MgO & $\mathrm{CaO}$ & $\mathrm{Na}_{2} \mathrm{O}$ & $\mathbf{K}_{2} \mathbf{O}$ & $\mathbf{P}_{2} \mathbf{O}_{5}$ & $\mathrm{SO}_{3}$ & Total \\
\hline 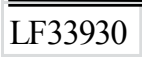 & 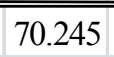 & 0.985 & 19.245 & 6.662 & $\overline{0.145}$ & 43 & 0.037 & 0.055 & 1.840 & .043 & 0.009 & 99. \\
\hline 1 & .865 & 3 & & 7 & 01 & 13 & 0.007 & 553 & 70 & 19 & 05 & 4 \\
\hline 933 & 73 & 0 & 24.122 & 613 & 0.078 & 58 & 0.038 & 66 & 2.379 & 031 & 15 & 00.21 \\
\hline 3 & 42 & & & & 22 & & 0. & 03 & 48 & 030 & & 99.96 \\
\hline 4 & & & & & 0.031 & & 0. & 67 & & 0.026 & & 37.00 \\
\hline 5 & & 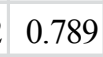 & & & 53 & & & 0.047 & & & & 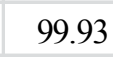 \\
\hline 6 & 592 & 0 & 2 & 0 & 41 & 38 & 0.205 & 56 & 58 & 60 & .024 & 9.87 \\
\hline 933 & 66.067 & 0.942 & 2 & & 0.037 & 0.518 & -0.009 & 0.032 & 78 & 047 & 004 & 99.9 \\
\hline 33 & .179 & 1 & 28.681 & & 0.017 & 0.448 & -0.047 & 0.022 & .067 & 0.054 & 0.005 & 100.39 \\
\hline 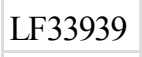 & & & & & & & 0. & 67 & 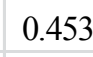 & 0.093 & & 99.57 \\
\hline 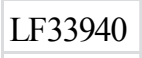 & & & & & & & -0 & & & & & 99.18 \\
\hline & & & & & & & & & & & & \\
\hline & & & & & & & & & & & & \\
\hline \multicolumn{13}{|c|}{ XRF Results for major oxides in recalculated regolith samples } \\
\hline ead & $\mathrm{iO}_{2}$ & $\mathrm{TiO}_{2}$ & $\mathbf{A l}_{2} \mathbf{O}_{3}$ & $\mathrm{Fe}_{2} \mathrm{O}_{3}$ & MnO & $\mathrm{MgO}$ & $\mathrm{CaO}$ & $\mathrm{Na}_{2} \mathrm{O}$ & $\mathbf{K}_{2} \mathbf{O}$ & $\mathbf{P}_{2} \mathbf{O}_{5}$ & $\mathrm{SO}_{3}$ & lotal \\
\hline 0 & & & & & & & & 0 & & - & & .11 \\
\hline & & & & & & & & & & & & 37.40 \\
\hline & & & & & 0.00 & & & 0 & & 0.000 & & 100.2 \\
\hline$F 33{ }^{6}$ & & 0.00 & 0.00 & 0 & 00 & 0 & 0. & 0.00 & 0.000 & 0.000 & 0.000 & 99.96 \\
\hline LF3 & & & & & & & & 00 & 00 & 00 & & 99.83 \\
\hline 53 & & & & & 0.000 & 0.00 & 0. & 0.00 & 0.000 & .000 & .000 & 99.93 \\
\hline 年 & & & & & & & & 00 & . & 00 & & 99.8 \\
\hline & & & & & & & & 0 & & & & 0.0 \\
\hline 5230 & & 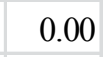 & 0.00 & & 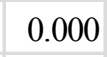 & 5 & 0 & 00 & 00 & 00 & .000 & 100.39 \\
\hline I 520 & & & & & & & & 0 & & 00 & & .57 \\
\hline$F 359$ & & 0. & & & 0.000 & 0.0 & 0. & 0.00 & 0.000 & 0.000 & 0.000 & 99.18 \\
\hline F33941 & 0.00 & 0.00 & 0.00 & 0.00 & 0.000 & 0.00 & 0.00 & 0.00 & 0.000 & 0.000 & 0.000 & 99.85 \\
\hline
\end{tabular}


Table 2. Calculated ratio of $\mathrm{K} / \mathrm{Al}$ and $\mathrm{Mg} / \mathrm{Al}$ from oxides in regolith samples from northern Ghana to aid in comparing two mobile elements with a relatively immobile element

\begin{tabular}{|c|c|c|c|c|c|c|c|c|}
\hline Sample ID & $\mathrm{MgO}(\%)$ & $\mathrm{Mg}$ & $\mathrm{Mg} / \mathrm{Al}$ & $\mathrm{K}_{2} \mathrm{O}(\%)$ & K & $\mathrm{K} / \mathrm{Al}$ & $\mathrm{A} 12 \mathrm{O} 3(\%)$ & $\mathrm{Al}$ \\
\hline FHA002 & 1.28 & 0.77 & 0.08 & 0.70 & 0.58 & 0.06 & 18.45 & 9.77 \\
\hline FHA008 & 1.38 & 0.83 & 0.08 & 0.98 & 0.81 & 0.08 & 19.07 & 10.10 \\
\hline FHA011 & 0.89 & 0.53 & 0.06 & 0.71 & 0.59 & 0.07 & 15.94 & 8.44 \\
\hline MPL009 & 1.77 & 1.06 & 0.11 & 1.86 & 1.54 & 0.16 & 17.99 & 9.52 \\
\hline MPL014 & 1.24 & 0.74 & 0.12 & 0.89 & 0.74 & 0.12 & 11.97 & 6.34 \\
\hline MPL019 & 0.84 & 0.50 & 0.06 & 0.42 & 0.35 & 0.04 & 16.30 & 8.63 \\
\hline RK006 & 1.20 & 0.72 & 0.07 & 0.91 & 0.75 & 0.07 & 19.98 & 10.58 \\
\hline RK010 & 0.60 & 0.36 & 0.05 & 0.54 & 0.45 & 0.06 & 14.28 & 7.56 \\
\hline RK016 & 1.00 & 0.60 & 0.08 & 0.71 & 0.59 & 0.07 & 14.92 & 7.90 \\
\hline SB002 & 2.10 & 1.26 & 0.09 & 1.91 & 1.58 & 0.12 & 25.49 & 13.49 \\
\hline SB003 & 2.08 & 1.25 & 0.09 & 1.90 & 1.58 & 0.11 & 27.36 & 14.48 \\
\hline SB004 & 1.95 & 1.17 & 0.10 & 1.63 & 1.35 & 0.11 & 22.79 & 12.07 \\
\hline EA001 & 0.41 & 0.25 & 0.03 & 1.72 & 1.43 & 0.15 & 17.96 & 9.51 \\
\hline EA002 & 0.48 & 0.29 & 0.03 & 2.20 & 1.82 & 0.16 & 21.49 & 11.38 \\
\hline EA003 & 0.70 & 0.42 & 0.04 & 2.20 & 1.82 & 0.15 & 22.35 & 11.83 \\
\hline EA004 & 0.64 & 0.38 & 0.05 & 1.74 & 1.44 & 0.19 & 14.30 & 7.57 \\
\hline EA005 & 0.81 & 0.49 & 0.05 & 2.01 & 1.67 & 0.17 & 19.06 & 10.09 \\
\hline EA006 & 2.12 & 1.27 & 0.12 & 2.39 & 1.98 & 0.19 & 20.10 & 10.64 \\
\hline EA007 & 0.45 & 0.27 & 0.04 & 1.63 & 1.35 & 0.18 & 14.26 & 7.55 \\
\hline EA008 & 0.48 & 0.29 & 0.03 & 1.93 & 1.60 & 0.15 & 20.34 & 10.77 \\
\hline EA009 & 0.41 & 0.25 & 0.02 & 1.88 & 1.56 & 0.11 & 26.12 & 13.83 \\
\hline EA010 & 0.19 & 0.11 & 0.03 & 0.43 & 0.36 & 0.08 & 8.27 & 4.38 \\
\hline EA011 & 0.18 & 0.11 & 0.01 & 0.20 & 0.17 & 0.01 & 21.37 & 11.31 \\
\hline EA012 & 0.16 & 0.10 & 0.01 & 0.18 & 0.15 & 0.01 & 26.19 & 13.86 \\
\hline A15 & 1.54 & 0.92 & 0.06 & 0.80 & 0.66 & 0.04 & 31.48 & 16.67 \\
\hline J19 & 1.24 & 0.74 & 0.07 & 0.64 & 0.53 & 0.05 & 21.27 & 11.26 \\
\hline $\mathrm{J} 20$ & 0.85 & 0.51 & 0.04 & 1.71 & 1.42 & 0.11 & 25.16 & 13.32 \\
\hline $\mathrm{J} 21$ & 1.25 & 0.75 & 0.09 & 0.91 & 0.75 & 0.09 & 16.64 & 8.81 \\
\hline $\mathrm{J} 23$ & 1.47 & 0.88 & 0.06 & 6.18 & 5.12 & 0.36 & 26.65 & 14.11 \\
\hline $\mathrm{J} 24$ & 0.81 & 0.49 & 0.04 & 0.61 & 0.51 & 0.04 & 22.74 & 12.04 \\
\hline $\mathrm{J} 25$ & 1.31 & 0.79 & 0.08 & 0.47 & 0.39 & 0.04 & 18.00 & 9.53 \\
\hline $\mathrm{J} 26$ & 1.28 & 0.77 & 0.06 & 0.54 & 0.45 & 0.04 & 23.00 & 12.18 \\
\hline $\mathrm{J} 27$ & 1.46 & 0.88 & 0.06 & 1.55 & 1.28 & 0.09 & 28.05 & 14.85 \\
\hline $\mathrm{J} 28$ & 1.36 & 0.82 & 0.10 & 0.93 & 0.77 & 0.10 & 14.70 & 7.78 \\
\hline K20 & 1.17 & 0.70 & 0.08 & 0.58 & 0.48 & 0.06 & 16.06 & 8.50 \\
\hline K21 & 1.50 & 0.90 & 0.09 & 1.88 & 1.56 & 0.16 & 18.51 & 9.80 \\
\hline K22 & 1.13 & 0.68 & 0.06 & 0.64 & 0.53 & 0.04 & 23.20 & 12.28 \\
\hline K23 & 1.15 & 0.69 & 0.04 & 0.44 & 0.36 & 0.02 & 29.69 & 15.72 \\
\hline K24 & 1.01 & 0.61 & 0.05 & 0.48 & 0.40 & 0.03 & 23.25 & 12.31 \\
\hline K26 & 1.35 & 0.81 & 0.04 & 0.33 & 0.27 & 0.01 & 38.25 & 20.25 \\
\hline K27 & 1.06 & 0.64 & 0.06 & 4.57 & 3.79 & 0.34 & 21.07 & 11.15 \\
\hline K28 & 0.93 & 0.56 & 0.03 & 2.49 & 2.06 & 0.11 & 33.97 & 17.98 \\
\hline L19 & 1.26 & 0.76 & 0.04 & 1.56 & 1.29 & 0.08 & 32.07 & 16.98 \\
\hline L20 & 1.32 & 0.79 & 0.16 & 2.39 & 1.98 & 0.40 & 9.44 & 5.00 \\
\hline
\end{tabular}




\begin{tabular}{|c|c|c|c|c|c|c|c|c|}
\hline L21 & 1.08 & 0.65 & 0.03 & 0.81 & 0.67 & 0.04 & 35.36 & 18.72 \\
\hline L22 & 1.13 & 0.68 & 0.05 & 0.32 & 0.27 & 0.02 & 25.34 & 13.41 \\
\hline $\mathrm{L} 23$ & 1.03 & 0.62 & 0.21 & 0.86 & 0.71 & 0.24 & 5.56 & 2.94 \\
\hline L24 & 1.45 & 0.87 & 0.05 & 0.53 & 0.44 & 0.02 & 35.26 & 18.67 \\
\hline L25 & 1.40 & 0.84 & 0.04 & 1.13 & 0.94 & 0.05 & 38.15 & 20.20 \\
\hline L26 & 1.26 & 0.76 & 0.06 & 0.87 & 0.72 & 0.06 & 23.94 & 12.67 \\
\hline L27 & 1.58 & 0.95 & 0.05 & 3.42 & 2.84 & 0.16 & 32.95 & 17.44 \\
\hline L28 & 1.67 & 1.00 & 0.09 & 2.99 & 2.48 & 0.21 & 22.13 & 11.72 \\
\hline M19 & 1.30 & 0.78 & 0.05 & 0.79 & 0.65 & 0.04 & 29.41 & 15.57 \\
\hline M20 & 1.29 & 0.77 & 0.04 & 0.27 & 0.22 & 0.01 & 37.99 & 20.11 \\
\hline M21 & 1.29 & 0.77 & 0.05 & 0.33 & 0.27 & 0.02 & 30.31 & 16.05 \\
\hline M22 & 1.23 & 0.74 & 0.06 & 0.92 & 0.76 & 0.06 & 24.84 & 13.15 \\
\hline M24 & 1.43 & 0.86 & 0.10 & 0.77 & 0.64 & 0.07 & 16.18 & 8.57 \\
\hline M25 & 1.41 & 0.85 & 0.07 & 0.91 & 0.75 & 0.06 & 23.43 & 12.40 \\
\hline M26 & 1.40 & 0.84 & 0.07 & 1.16 & 0.96 & 0.08 & 22.18 & 11.74 \\
\hline M27 & 1.18 & 0.71 & 0.05 & 1.09 & 0.90 & 0.07 & 24.93 & 13.20 \\
\hline M28 & 1.58 & 0.95 & 0.05 & 1.24 & 1.03 & 0.05 & 38.68 & 20.48 \\
\hline N19 & 1.42 & 0.85 & 0.04 & 1.03 & 0.85 & 0.04 & 36.53 & 19.34 \\
\hline N20 & 1.00 & 0.60 & 0.21 & 1.14 & 0.95 & 0.34 & 5.28 & 2.80 \\
\hline N21 & 1.29 & 0.77 & 0.06 & 1.13 & 0.94 & 0.08 & 22.51 & 11.92 \\
\hline N22 & 0.98 & 0.59 & 0.04 & 0.44 & 0.36 & 0.03 & 26.96 & 14.27 \\
\hline $\mathrm{N} 24$ & 4.50 & 2.70 & 0.22 & 1.76 & 1.46 & 0.12 & 23.23 & 12.30 \\
\hline $\mathrm{N} 25$ & 2.44 & 1.46 & 0.07 & 1.17 & 0.97 & 0.05 & 39.21 & 20.76 \\
\hline N26 & 2.18 & 1.31 & 0.08 & 1.10 & 0.91 & 0.06 & 30.64 & 16.22 \\
\hline $\mathrm{N} 27$ & 1.28 & 0.77 & 0.05 & 0.90 & 0.75 & 0.05 & 29.66 & 15.70 \\
\hline $\mathrm{N} 28$ & 1.29 & 0.77 & 0.05 & 0.96 & 0.80 & 0.05 & 28.51 & 15.09 \\
\hline O19 & 1.12 & 0.67 & 0.03 & 0.29 & 0.24 & 0.01 & 37.81 & 20.02 \\
\hline $\mathrm{O} 20$ & 0.84 & 0.50 & 0.05 & 0.49 & 0.41 & 0.04 & 18.73 & 9.92 \\
\hline $\mathrm{O} 22$ & 1.07 & 0.64 & 0.06 & 0.98 & 0.81 & 0.08 & 18.77 & 9.94 \\
\hline $\mathrm{O} 23$ & 1.22 & 0.73 & 0.04 & 2.02 & 1.67 & 0.09 & 36.32 & 19.23 \\
\hline $\mathrm{O} 24$ & 1.16 & 0.70 & 0.06 & 6.08 & 5.04 & 0.40 & 23.81 & 12.61 \\
\hline $\mathrm{O} 26$ & 1.17 & 0.70 & 0.03 & 0.70 & 0.58 & 0.03 & 40.22 & 21.29 \\
\hline $\mathrm{O} 27$ & 2.34 & 1.40 & 0.11 & 1.16 & 0.96 & 0.07 & 24.67 & 13.06 \\
\hline P19 & 1.39 & 0.83 & 0.05 & 0.74 & 0.61 & 0.04 & 30.70 & 16.25 \\
\hline $\mathrm{P} 20$ & 2.42 & 1.45 & 0.08 & 2.37 & 1.96 & 0.11 & 34.93 & 18.49 \\
\hline $\mathrm{P} 21$ & 1.28 & 0.77 & 0.06 & 1.35 & 1.12 & 0.08 & 25.08 & 13.28 \\
\hline $\mathrm{P} 22$ & 1.18 & 0.71 & 0.04 & 0.93 & 0.77 & 0.04 & 34.30 & 18.16 \\
\hline $\mathrm{P} 23$ & 1.41 & 0.85 & 0.05 & 0.58 & 0.48 & 0.03 & 31.62 & 16.74 \\
\hline P24 & 1.06 & 0.64 & 0.04 & 2.74 & 2.27 & 0.14 & 31.70 & 16.78 \\
\hline $\mathrm{P} 25$ & 1.10 & 0.66 & 0.05 & 1.24 & 1.03 & 0.08 & 23.56 & 12.47 \\
\hline P26 & 1.10 & 0.66 & 0.06 & 1.07 & 0.89 & 0.08 & 22.20 & 11.75 \\
\hline P27 & 1.12 & 0.67 & 0.06 & 0.72 & 0.60 & 0.05 & 21.77 & 11.53 \\
\hline $\mathrm{P} 28$ & 1.00 & 0.60 & 0.17 & 0.42 & 0.35 & 0.10 & 6.77 & 3.58 \\
\hline Q20 & 0.98 & 0.59 & 0.06 & 0.83 & 0.69 & 0.07 & 17.44 & 9.23 \\
\hline Q21 & 1.24 & 0.74 & 0.05 & 0.94 & 0.78 & 0.05 & 27.65 & 14.64 \\
\hline Q22 & 1.08 & 0.65 & 0.04 & 0.41 & 0.34 & 0.02 & 33.34 & 17.65 \\
\hline Q23 & 1.30 & 0.78 & 0.12 & 1.68 & 1.39 & 0.21 & 12.55 & 6.64 \\
\hline
\end{tabular}




\begin{tabular}{|c|c|c|c|c|c|c|c|c|}
\hline Q24 & 1.62 & 0.97 & 0.05 & 1.93 & 1.60 & 0.09 & 33.54 & 17.76 \\
\hline Q25 & 1.14 & 0.68 & 0.05 & 1.32 & 1.09 & 0.08 & 24.70 & 13.08 \\
\hline Q26 & 1.24 & 0.74 & 0.05 & 0.93 & 0.77 & 0.05 & 31.18 & 16.51 \\
\hline Q27 & 1.41 & 0.85 & 0.10 & 0.35 & 0.29 & 0.03 & 16.37 & 8.67 \\
\hline Q28 & 1.35 & 0.81 & 0.10 & 0.67 & 0.56 & 0.07 & 14.87 & 7.87 \\
\hline R19 & 1.11 & 0.67 & 0.07 & 1.80 & 1.49 & 0.15 & 18.40 & 9.74 \\
\hline $\mathrm{R} 20$ & 1.40 & 0.84 & 0.04 & 0.65 & 0.54 & 0.03 & 37.13 & 19.66 \\
\hline $\mathrm{R} 21$ & 1.50 & 0.90 & 0.06 & 0.56 & 0.46 & 0.03 & 30.43 & 16.11 \\
\hline $\mathrm{R} 22$ & 1.53 & 0.92 & 0.05 & 1.30 & 1.08 & 0.05 & 38.52 & 20.39 \\
\hline $\mathrm{R} 23$ & 1.01 & 0.61 & 0.04 & 0.69 & 0.57 & 0.03 & 31.41 & 16.63 \\
\hline $\mathrm{R} 24$ & 1.16 & 0.70 & 0.04 & 2.37 & 1.96 & 0.13 & 29.65 & 15.70 \\
\hline $\mathrm{R} 25$ & 1.51 & 0.91 & 0.05 & 1.05 & 0.87 & 0.05 & 34.91 & 18.48 \\
\hline $\mathrm{R} 26$ & 1.57 & 0.94 & 0.07 & 2.30 & 1.91 & 0.14 & 25.90 & 13.71 \\
\hline $\mathrm{R} 27$ & 1.23 & 0.74 & 0.05 & 1.12 & 0.93 & 0.07 & 26.78 & 14.18 \\
\hline R28 & 1.38 & 0.83 & 0.24 & 0.31 & 0.26 & 0.08 & 6.42 & 3.40 \\
\hline S19 & 1.31 & 0.79 & 0.06 & 1.27 & 1.05 & 0.08 & 23.74 & 12.57 \\
\hline $\mathrm{S} 20$ & 1.49 & 0.89 & 0.07 & 0.70 & 0.58 & 0.05 & 22.95 & 12.15 \\
\hline S21 & 1.51 & 0.91 & 0.14 & 3.11 & 2.58 & 0.39 & 12.34 & 6.53 \\
\hline $\mathrm{S} 22$ & 0.91 & 0.55 & 0.07 & 1.06 & 0.88 & 0.11 & 15.10 & 7.99 \\
\hline $\mathrm{S} 23$ & 1.34 & 0.80 & 0.08 & 1.99 & 1.65 & 0.16 & 19.53 & 10.34 \\
\hline S24 & 1.39 & 0.83 & 0.04 & 0.64 & 0.53 & 0.03 & 39.19 & 20.75 \\
\hline $\mathrm{S} 25$ & 1.32 & 0.79 & 0.05 & 3.38 & 2.80 & 0.16 & 32.65 & 17.28 \\
\hline S26 & 1.22 & 0.73 & 0.05 & 0.76 & 0.63 & 0.04 & 28.39 & 15.03 \\
\hline S28 & 1.28 & 0.77 & 0.05 & 1.26 & 1.04 & 0.07 & 28.69 & 15.19 \\
\hline $\mathrm{T} 23$ & 1.31 & 0.79 & 0.07 & 0.66 & 0.55 & 0.05 & 22.11 & 11.71 \\
\hline U19 & 1.43 & 0.86 & 0.05 & 1.38 & 1.14 & 0.06 & 33.32 & 17.64 \\
\hline $\mathrm{A} 01$ & 0.95 & 0.57 & 0.06 & 0.54 & 0.45 & 0.05 & 17.77 & 9.41 \\
\hline A08 & 1.83 & 1.10 & 0.06 & 0.89 & 0.74 & 0.04 & 35.97 & 19.04 \\
\hline A09 & 3.24 & 1.94 & 0.11 & 1.35 & 1.12 & 0.06 & 34.18 & 18.09 \\
\hline $\mathrm{A} 10$ & 3.56 & 2.14 & 0.11 & 0.97 & 0.80 & 0.04 & 37.39 & 19.79 \\
\hline A11 & 2.75 & 1.65 & 0.15 & 1.30 & 1.08 & 0.10 & 21.42 & 11.34 \\
\hline A12 & 2.87 & 1.72 & 0.08 & 1.29 & 1.07 & 0.05 & 38.57 & 20.42 \\
\hline A13 & 1.71 & 1.03 & 0.07 & 0.97 & 0.80 & 0.05 & 29.69 & 15.72 \\
\hline A14 & 1.54 & 0.92 & 0.05 & 0.78 & 0.65 & 0.03 & 37.47 & 19.84 \\
\hline A15 & 1.58 & 0.95 & 0.05 & 1.41 & 1.17 & 0.06 & 37.30 & 19.75 \\
\hline A16 & 1.48 & 0.89 & 0.04 & 1.35 & 1.12 & 0.06 & 38.39 & 20.32 \\
\hline A17 & 1.61 & 0.97 & 0.05 & 1.23 & 1.02 & 0.05 & 37.00 & 19.59 \\
\hline A18 & 1.71 & 1.03 & 0.06 & 1.77 & 1.47 & 0.08 & 33.34 & 17.65 \\
\hline A 20 & 1.53 & 0.92 & 0.04 & 1.41 & 1.17 & 0.06 & 38.77 & 20.52 \\
\hline $\mathrm{A} 21$ & 1.86 & 1.12 & 0.11 & 3.07 & 2.55 & 0.25 & 19.44 & 10.29 \\
\hline A22 & 1.24 & 0.74 & 0.11 & 0.78 & 0.65 & 0.10 & 12.70 & 6.72 \\
\hline A 23 & 1.50 & 0.90 & 0.05 & 1.03 & 0.85 & 0.05 & 33.49 & 17.73 \\
\hline A24 & 1.43 & 0.86 & 0.07 & 1.45 & 1.20 & 0.10 & 21.80 & 11.54 \\
\hline A25 & 1.54 & 0.92 & 0.05 & 0.89 & 0.74 & 0.04 & 34.76 & 18.40 \\
\hline A26 & 1.34 & 0.80 & 0.06 & 0.47 & 0.39 & 0.03 & 27.20 & 14.40 \\
\hline A 27 & 1.45 & 0.87 & 0.07 & 1.03 & 0.85 & 0.07 & 23.45 & 12.41 \\
\hline
\end{tabular}




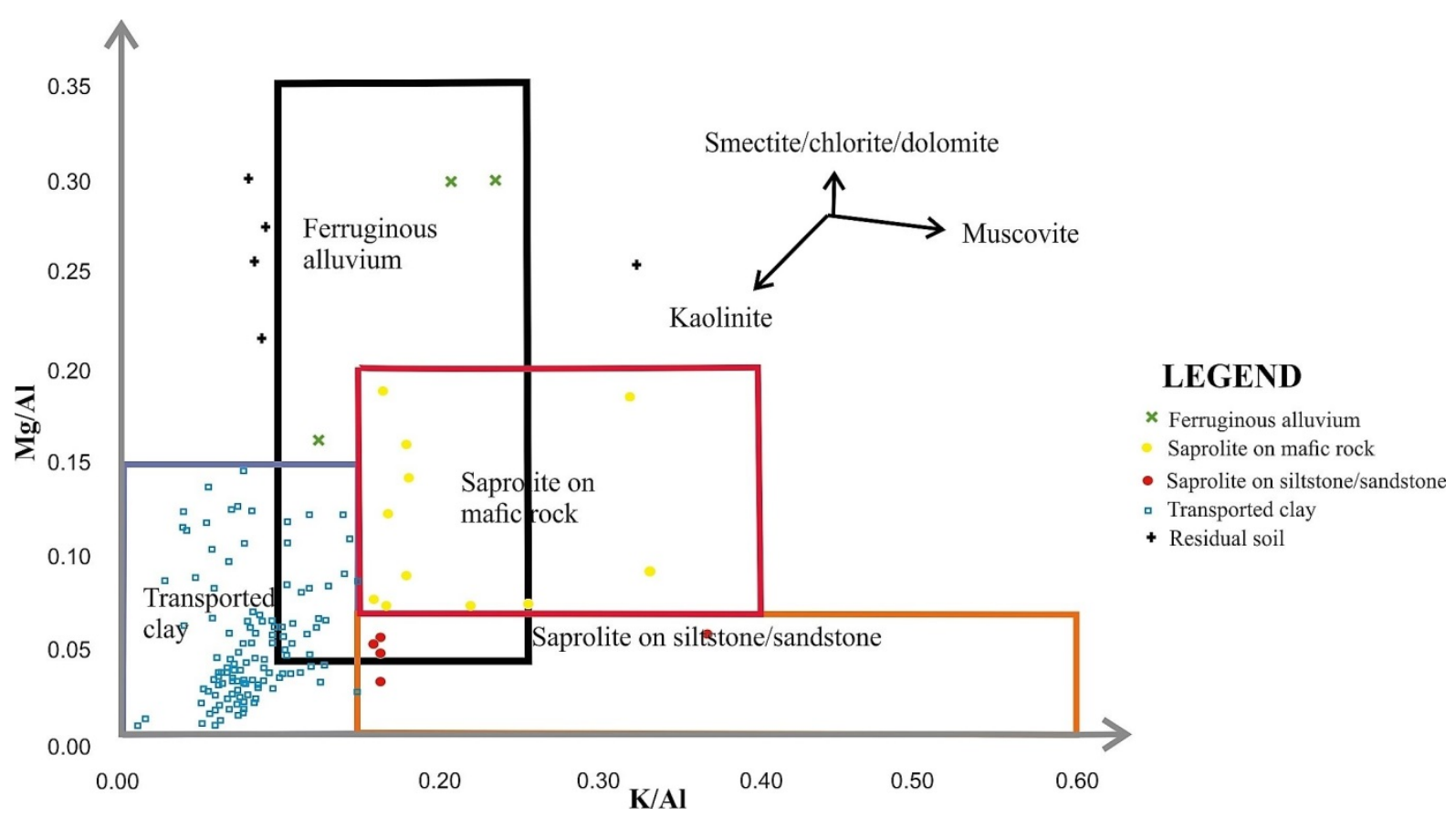

Figure 8. $\mathrm{Mg} / \mathrm{Al}$ vs. $\mathrm{K} / \mathrm{Al}$ ratio plot (wt. \% proportion) from four wide-ranging regolith units (Arhin and Zango, 2015)

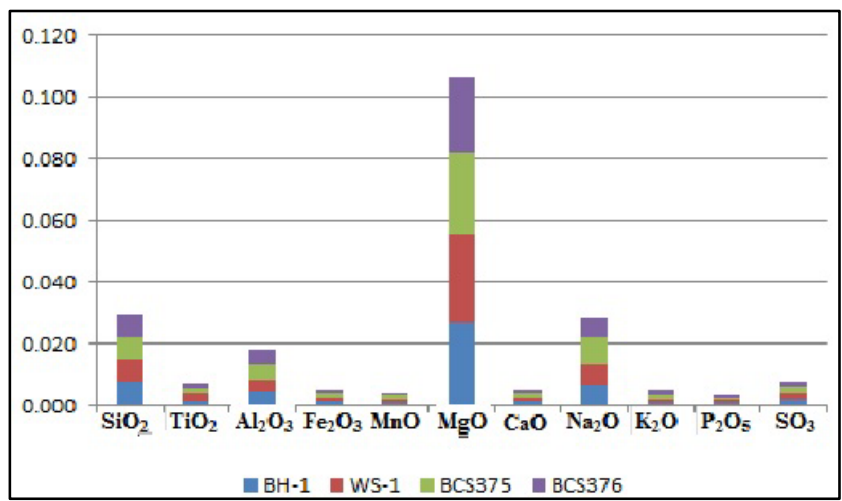

A. Major oxides comparisons from sub samples taken from a standard reference material

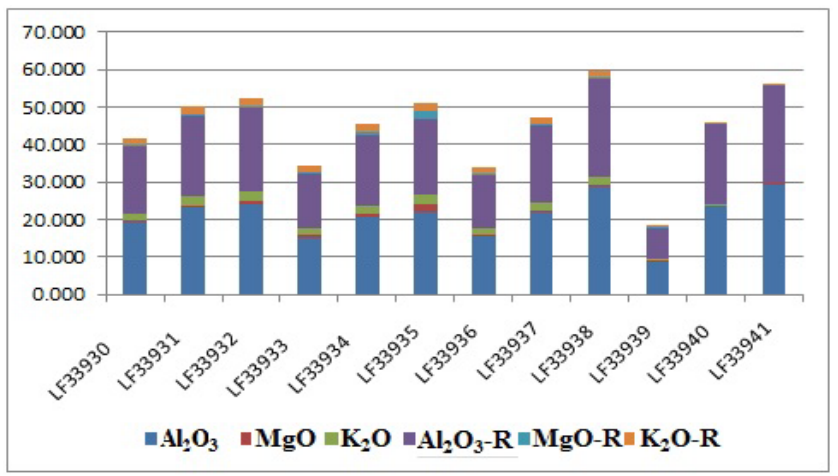

B. Major oxide concentrations in original and recalculated field samples

Figure 9. Comparisons of standard reference inserted samples, original and recalculated major element concentrations of field samples for the evaluation of the quality of the analysis

\section{Discussion}

The regolith map developed for the area confirmed the complexity of the surface environment suggesting geochemical responses developed in the transformed surfaces due to regolith-landform evolution processes may vary. With these differences in regolith materials haphazardly distributed at the surface environment; improper characterization and classification of the regolith will lead to challenging surface data interpretations and these can influence metal transports and their concentrations $[2,3]$. As the mineral anomaly detection of world-class mineral deposits continues to decline, focus on geochemical exploration methods designed for regolith-dominated terrains should distinguish the landscape on the basis of regolith types before anomaly delineation. But the common characteristics of most areas in the study is characterized by deep weathering, modified to varying degrees of leaching and erosion of which some of the upper landscapes show subsequent depositional features. Characterizing the resultant landforms after the landscape evolution processes triggered generally by climate are broadly similar and thin veneers of soils overlying terrains classified into ferruginous, relict, erosional and depositional. Distinguishing the landscape with respect to the regolith type as seen in Figure 7; 50 samples were collected from ferruginous regolith that have an ability to conceal the anomaly from detection [4]; 32 samples were collected from the relict, which $[2,3,17]$ have transformed and retained geochemical signatures from the fractionation of the bedrock mineralization representing residual anomaly; 8 samples were taken from the erosional 
regolith and these may have bedrock mineralization if associated with underlying mineralization [3] and 64 samples were taken from depositional regimes whose geochemical expressions may or may not represent the sampled terrain [2]. These classifications were based on field descriptions and may be fraught with challenges where features that define the type of regolith are not well demonstrated. The irregular medley of regolith materials mapped in the area and the general assertion that quality of mapped regolith is scale dependent [1,18] suggest further quantitative characterization of regolith materials using geochemistry as a surrogate for mineralogy to support the qualitative description and characterization of mapped regolith will be improved. The probe into the accuracy of the field characterized regolith was quizzed out by comparing regolith material types from the plot of $\mathrm{Mg} / \mathrm{Al}$ and $\mathrm{K} / \mathrm{Al}$. Some disparities were identified between the qualitative and the quantitative regolith identification and characterization. These however were not strange because the scale of the field regolith mapping was regional scale (i.e. 1: 50, 000) and that there are some misleading classification of some regolith materials mapped in Africa $[1,5,6]$. From Fig. 8 the characterization and identification of regolith based on the four indices ferruginous, relict, erosional and depositional regolith were 10,11 and 123 respectively. The error margins with superposition of the field regolith mapping data and $\mathrm{Mg} / \mathrm{Al}$ vs. K/Al index plot vary when Figs. 3 and 9 were analyzed and compared. $80 \%$ of the field mapped regolith was not wholly ferruginous units. Similarly $75 \%$ of mapped relict materials, $38 \%$ of erosional and $92 \%$ of depositional regolith materials were inaccurate. This confirms [1-3] observations that the superficial regolith materials expressed at the upper portions of the landscapes will assume broad regolith classes to have developed from a simple weathering history processes whereas in actuality they may be an expression of cumulative effects of long complex weathering histories. This do not suggests that field regolith mapping is not effective in characterizing the regolith but because the scale of mapping was too large for the overall extrapolations for areas not visited accounted for the errors observed between the two regolith characterization processes.

Therefore supporting the field regolith-landform mapping the ratio plots of $\mathrm{Mg} / \mathrm{Al}$ and $\mathrm{K} / \mathrm{Al}$ will provide better characterization and identification of the different sample materials because the footprint of bedrock geochemistry will be reflective in the overlying regolith if in-situ and vice versa $[7,17,19,20]$. Regolith landform unit classifications are carried out and generally are made to address specific problems and fit for purpose. If the purpose of the identification and characterization is for mineral exploration purposes residual, transported and modified regolith areas can be distinguished and challenges of surface geochemical data interpretation confronting many exploration companies following up 'false' geochemical anomalies can be avoided whilst 'real' geochemical signatures can be defined for follow up surveys. Conversely, if the regolith-landform unit classification is meant for agricultural, engineering or environmental planning purposes the correct terrain can be selected on the basis of the precise and formal regolith information. Hence, assuming the distinction between residual, transported and modified regolith-landform surfaces impede on the smooth interpretation of surface geochemical data and further poses challenges for mineral exploration surveys where 'false' and 'real' anomalies needs to be distinguish for the avoidance of following only real geochemical anomalies then the application of $\mathrm{Mg} / \mathrm{Al}$ and $\mathrm{K} / \mathrm{Al}$ plot to unravel the different regolith units should be considered. This may not be too expensive as many of the mining and mineral exploration companies have huge multi-element dataset that can be used for this purpose to create an additional layer to be used alongside the regolith maps developed from the regolith field mapping information to aid in the surface geochemical results interpretations.

The assurance that the data obtained for the ratio plots were good and acceptable for the regolith identification and indexing work was demonstrated by quality control analysis presented in Figure 9. Figure 9 shows good correlation except $\mathrm{MgO}$ which were inconstant in some of the field samples but the weight \% in the sub samples inserted in the batch of samples submitted to the laboratories returned consistent values. Though there was some variations in $\mathrm{MgO}$ contents in the field samples but the margin of variations were insignificant to impact on the quality of the analytical method hence the acceptance of the UXRF results. Using regolith geochemistry as a surrogate to regolith mineralogy because of the fineness of the sampled regolith materials; the regolith types of the sampled materials were revealed. The semi-quantitative geochemical data confirmed the correctness of some of the field described regolith materials. But there were some that the field identified regolith classes were at variance based on the index plot results. But from Nude and Arhin [21] the regolith geochemical data should reflect the underlying substrate if in situ and incomparable with the underlying substrate if transported. Conversely low K/Al and low $\mathrm{Mg} / \mathrm{Al}$ ratios reflect sediments of diverse origins whilst low $\mathrm{K} / \mathrm{Al}$ but high $\mathrm{Mg} / \mathrm{Al}$ depicts environments covered by relict and semi residual regolith units. Terrains marked by saprolite are characterized by relatively high $\mathrm{K} / \mathrm{Al}$ and moderate $\mathrm{Mg} / \mathrm{Al}$ ratios. Where adequate major oxide data are available, the method can be used to authenticate the field regolith descriptions before geochemical data interpretations. However, in absolute values terms as seen from Fig. $8 ; \mathrm{Mg} / \mathrm{Al}$ value for transported regolith material and saprolite on mafic rock should be in the limits of $0.00-0.15$ and $0.7-0.2$ respectively whiles that of $\mathrm{K} / \mathrm{Al}$ should be in the range of $0.00-0.16$ and $0.16-0.4$ respectively. The relict materials will lay between $\geq 0.15$ 
$\mathrm{Mg} / \mathrm{Al}$ and $\leq 0.15 \mathrm{~K} / \mathrm{Al}$ ratios. The ferruginous materials will lie in these ranges $0.05-0.35$ for $\mathrm{Mg} / \mathrm{Al}$ and $0.15-0.28$ for $\mathrm{K} / \mathrm{Al}$ respectively. But because ferruginous materials can form over residual and transported weathered materials further probe may be required to distinguish them.

\section{Conclusions}

The study acknowledged some misleading classification of field classified regolith units and agree with Arhin et al. [1] that the surface regolith in the areas have undergone heterogeneous mixing as a result of the past and present regolith landform evolutional events. In addition the following conclusions can be drawn from the present study:

- The field regolith mapping accuracy depends on scale use and thus to develop more precise regolith maps fit for purpose; a prospect scale (e.g. 1: 500 or smaller) should be used

- $\quad$ The ratio plot of $\mathrm{Mg} / \mathrm{Al}$ and $\mathrm{K} / \mathrm{Al}$ recognized most of the samples collected for the study were made up of transported clays/sediments deposited at the present surface to have originated and come from diverse sources.

- The varying regolith mapping material distributions on the landscape identified from the 154 samples enabled the characterization and identification of the different sample materials because an overprint of bedrock geochemistry was reflective in the residual overlying regolith but unrelated with regolith materials whose origins bears no relationship to the underlying bedrock.

- That the ratio plot identification and characterization of the regolith unit type is site specific and only refer to the samples under investigation but can be tied to their geographic or landscape locations and

- In this study the plot of $\mathrm{Mg} / \mathrm{Al}$ and $\mathrm{K} / \mathrm{Al}$ was able to highlight the compositional variability of the regolith samples.

In conclusion the study recognized the ratio plots of $\mathrm{Mg} / \mathrm{Al}$ versus $\mathrm{K} / \mathrm{Al}$ as useful in supporting field identification of regolith-landform units (RLU) and suggest that $\mathrm{Mg} / \mathrm{Al}$ values for transported regolith material and saprolite on mafic rock should be in the limits of $0.00-0.15$ and $0.7-0.2$ whilst $\mathrm{K} / \mathrm{Al}$ ratio values in the range of 0.00-0.16 and 0.16-0.4 characterize the transported and erosional regoliths. The relict materials will fall between $\geq$ $0.15 \mathrm{Mg} / \mathrm{Al}$ and $\leq 0.15 \mathrm{~K} / \mathrm{Al}$ ratio values. The ferruginous materials will also be found between the zones $0.05-0.35$ for $\mathrm{Mg} / \mathrm{Al}$ and $0.15-0.28$ for $\mathrm{K} / \mathrm{Al}$. The authors conclude that the use of regolith geochemistry as surrogate to regolith mineralogy unstitch the various regolith material types in complex regolith terrains and thus it should be used in regolith type identifications in complex regolith terrains.

\section{Acknowledgements}

This work was supported by WAXI/AMIRA International (grant number P934A) and the authors wish to thank them profusely for their financial support. Also any other person(s) that reviewed the manuscript we say thank you. Universities of Leicester, UK and University for Development Studies, Ghana are also acknowledged. The authors really appreciate your contributions and assistance in whatever form. Support in kind by Azumah Resources Company; the exploration company working in the area is greatly appreciated.

\section{REFERENCES}

[1] Arhin, E., Jenkin, G.R.T., Cunningham, D, and Nude, P. 2015. Regolith mapping of deeply weathered terrain in savannah regions of the Birimian Lawra Greenstone Belt, Ghana. Journal of Geochemical Exploration, vol. 159, 194-207.

[2] Arhin E. and Nude P. M. 2009. Significance of regolith mapping and its implication for gold exploration in northern Ghana: a case study at Tinga and Kunche. Geochemistry: Exploration, Environment, Analysis, vol. 9, p. 63-69.

[3] Anand, R. R., Wildman, J.E., Varga, Z. S. and Phang, C., 2001. Regolith evolution and geochemical dispersion in transported and residual regolith-Bronzewing gold deposit: Geochemistry: Exploration, Environment, Analysis, vol. 1 (12), p. 256-276.

[4] Butt, C. R. M., and Zeegers, H. 1992. Regolith Exploration in Tropical and Subtropical Terrains: Handbook of Exploration Geochemistry 4. Elsevier, Amsterdam, 607 p.

[5] Bolster, S. J. S. 1999. Regolith mapping: is it really necessary? In: Ho, S. E., Geffress, G., and Davies, B. (Eds) Exploration geochemistry for the new millennium. Bulletin 30, Australian Institute of Geoscientists, Perth, p. 125-135.

[6] Butt, C. R. M., and Bristow, A. P., 2013. Relief inversion in the geomorphological evolution of sub-Saharan West Africa: Geomorphology, vol. 185, p. 16-26.

[7] Arhin, E and Zango, S.M. 2015. Unravelling regolith material types using $\mathrm{Mg} / \mathrm{Al}$ and $\mathrm{K} / \mathrm{Al}$ plot to support field regolith identification in the savannah regions of NW Ghana, West Africa, Journal of African Earth Sciences, vol. 112,p. 597-607/ Elsevier Publication.

[8] Dickson, K. B., Benneh, G. A., 1995. A New Geography of Ghana. $3^{\text {rd }}$ Revised Edition. Longman.

[9] Freyssinet, P., Butt, C. R. M., Morris, R. C., and Piantone, P., 2005. Ore-forming processes related to lateritic weathering. In: Hedenquist, J. W., Thomson, J. F. H., Goldfarb, R. J., Richards, J. P. (Eds), Economic Geology $100^{\text {th }}$ Anniversary Volume. Economic Geology Publishing Company, New Haven, Connecticut, p. 681-722. 
[10] Oberthuer, T., Vetter, U., Davis, D. W., and Amanor, J.A., 1998. Age constraints on gold mineralisation and Paleo Proterozoic crustal evolution in the Ashanti belt of southern Ghana. Precambrian Research, vol. 89 (3/4), p. 129-143.

[11] Egal, E., Thieblemont, D., Lahondere, D., Guerrot, C., Costea, C.A., Iliescu, D., Delor, C.,Goujou, J.C., Lafon, J.M., and Tegyey, M., 2002. Late Eburnean granitization and tectonics along the western and north-western margin of the Archean Kenema-Man domain (Guinea, West African Craton). Precambrian Research, vol. 117 (1/2), p. 57-84.

[12] Gasquet, D., Barbey, P., Adou, M., and Paquette, J. L., 2003. Structure, Sr-Nd isotope geochemistry and zircon $\mathrm{U}-\mathrm{Pb}$ geochronology of the granitoids of the Dabakala area (Cote d'Ivoire): evidence for a $2.3 \mathrm{Ga}$ crustal growth event in the Palaeoproterozoic of West Africa? Precambrian Research, vol. 127 (4), p. 329-354.

[13] Naba, S., Lompo, M., Debat P., Bouchez, J. L., and Beziat, D., 2004. Structure and emplacement model for late-orogenic Paleo Proterozoic granitoids: the Tenkodogo-Yamba elongate pluton (Eastern Burkina Faso). Journal of African Earth Science, vol. 38 (1), p. 41-57.

[14] Feybesse, J. L., Billa, M., Guerrot, C., Duguey, E., Lescuyer, J.-L., Milési, J. P., and Bouchot, V. 2006. The paleo Proterozoic Ghanaian province: geodynamic model and ore controls, including regional stress modelling. Precambrian Research, vol. 149 (3/4), p. 149-196.

[15] Baratoux, L., Matelka, V., Naba, S., Jessell, M. W., Grégoire, M., and Ganne, J., 2011, Juvenile Palaeoproterozoic crust evolution during the Eburnean orogeny $(\sim 2.2-2.0 \quad$ Ga $)$, Western Burkina Faso. Precambrian Research, vol. 191, p. 18-45.

[16] De Kock, G. S., Armstrong, R. A., Siegfried, H. P., and Thomas, E., 2011. Geochronology of the Birimian Super group of the West African Craton in the Wa-Bole region of west-central Ghana: implications for the stratigraphic framework. Journal of African Earth Sciences, vol. 59, p. 1-40, doi: 10.1016/j.afrearsci.2010.08.001, p. 291-294

[17] McQueen, K. G., 2004. The nature, origin and exploration significance of the regolith, Girilambone-Cobar region. In: McQueen, K. G., and Scott, K. M. (Eds), Exploration Field Workshop Cobar Region 2004 Proceedings, CRC LEME, Perth, p. 51-56.

[18] Anand, R. R and Paine, M. 2002. Regolith geology of the Yilgarn Craton, Western Australia: implications for exploration, Australian Journal of Earth Science, vol. 49, p. 3-163.

[19] McQueen, K. G., 2006. Unravelling the regolith with geochemistry. In Regolith 2006 - Consolidation and Dispersion of ideas. (Eds R. W., Fitzpatrick and P. Shand) p. 230 - 235. CRC LEME, Perth.

[20] McQueen, K. G., and McRae, A., 2004. New ways to explore through the regolith in western New South Wales. In: Pacrim 2004 congress, Proceedings, p. 232-238. The Australian Institute of Mining and Metallurgy, Melbourne.

[21] Nude \& Arhin 2009. Overbank sediments as appropriate geochemical sample media in regional sediment surveys. Journal of Geochemical Exploration, vol. 103, p. 50-56. 